\title{
Flutuação populacional circanual de coleópteros em granja avícola, em Pelotas, RS, Brasil ${ }^{1}$
}

\author{
Carla de Lima Bicho² Lúcia Massutti de Almeida $^{3}$, Paulo Bretanha Ribeiro ${ }^{4}$ \& Paulo Silveira Júnior ${ }^{5}$
}

1. Parte da Tese de Doutorado do autor sênior, Curso de Pós-Graduação em Ciências Biológicas, Área de Concentração em Entomologia, Universidade Federal do Paraná (UFPR). Contribuição nº 1422 do Departamento de Zoologia, UFPR.

2. Centro Federal de Educação Tecnológica de Pelotas (CEFET/RS), 96015-360 Pelotas, RS, Brasil. (clbicho@ufpel.tche.br)

3. Departamento de Zoologia, Setor de Ciências Biológicas, UFPR, Caixa Postal 19020, 81531-980 Curitiba, PR, Brasil. (lalmeida@ufpr.br)

4. Departamento de Microbiologia e Parasitologia, Instituto de Biologia, Universidade Federal de Pelotas (UFPel), Caixa Postal 354, 96010-900 Pelotas, RS, Brasil. (bretanha@ufpel.tche.br)

5. Departamento de Matemática, Estatística e Computação, Instituto de Física e Matemática, UFPel. (prpd@ufpel.tche.br)

\begin{abstract}
Fluctuation of Coleoptera population in poultry house, in Pelotas, RS, Brazil. The population fluctuation of Coleoptera in poultry house "Conjunto Agrotécnico Visconde da Graça" in Pelotas, Rio Grande do Sul, Brazil is investigated. Six collection methods were utilized: 1 ( 0 to 7 day-old chicken feces); 2 (7 to 14 day-old feces); 3 (14 to 21 day-old feces); 4 ( 0 to 21 dayold feces), 5 (accumulated feces); and 6 (tube trap). An analysis of polynomial regression was accomplished independently of the collection methods. The survey was conducted from August 1998 to July 1999. A total of 12,449 Coleoptera were collected, represented by the following species: Carcinops troglodytes (Paykull, 1811) (Histeridae) (6,444); Alphitobius diaperinus (Panzer, 1797) (Tenebrionidae) (2,896); Somotrichus unifasciatus (Dejean, 1792) (Carabidae) (1,190); Gnathocerus cornutus (Fabricius, 1798) (Tenebrionidae) (947); Euxestus sp. (Cerylonidae) (394); Euspilotus rubriculus (Marseul, 1855) (Histeridae) (213); Ontholestes sp. (Staphylinidae) (190); Dactylosternum sp. (Hydrophilidae) (93); Mezium americanum (Laporte, 1840) (Ptinidae) (43); Palorus subdepressus (Wollaston, 1864) (Tenebrionidae) (27); Tenebroides mauritanicus (L., 1758) (Trogossitidae) (8); Xyleborus ferrugineus (Fabricius, 1801) (Scolytidae) (1); and unidentified specimens of Dermestidae (3). The highest number of species was collected during March and July, and the smallest in September. The highest abundance was recorded in March $(2,159)$, while the lowest index of capture was noted in October (633). The population fluctuation was estimated for C. troglodytes, A. diaperinus, S. unifasciatus, G. cornutus, Euxestus sp., E. rubriculus, Ontholestes sp. and Dactylosternum sp.
\end{abstract}

KEYWORDS. Coleoptera, population fluctuation, poultry house.

RESUMO. Com o objetivo de conhecer a flutuação populacional de coleópteros na granja de aves do Conjunto Agrotécnico Visconde da Graça, em Pelotas, RS, foram utilizados seis métodos de coleta: 1 (fezes de galinhas com 0 a 7 dias); 2 (7 a 14 dias); 3 (14 a 21 dias); 4 (0 a 21 dias); 5 (fezes acumuladas) e 6 (armadilhas de tubo). Análises de regressão polinomial foram realizadas independentemente dos métodos de coleta. O estudo foi realizado de agosto de 1998 a julho de 1999. Foram capturados 12.449 coleópteros representados pelas seguintes espécies Carcinops troglodytes (Paykull, 1811) (Histeridae) (6.444); Alphitobius diaperinus (Panzer, 1797) (Tenebrionidae) (2.896); Somotrichus unifasciatus (Dejean, 1792) (Carabidae) (1.190); Gnathocerus cornutus (Fabricius, 1798) (Tenebrionidae) (947); Euxestus sp. (Cerylonidae) (394); Euspilotus rubriculus (Marseul, 1855) (Histeridae) (213); Ontholestes sp. (Staphylinidae) (190); Dactylosternum sp. (Hydrophilidae) (93); Mezium americanum (Laporte, 1840) (Ptinidae) (43); Palorus subdepressus (Wollaston, 1864) (Tenebrionidae) (27); Tenebroides mauritanicus (L., 1758) (Trogossitidae) (8); Xyleborus ferrugineus (Fabricius, 1801) (Scolytidae) (1); e espécimens não identificados de Dermestidae (3). O maior número de espécies ocorreu nos meses de março e julho e o menor, no mês de setembro. Em março foi registrada a maior abundância de coleópteros (2.159), enquanto que o menor índice de captura foi assinalado em outubro (633). A flutuação populacional foi estimada para C. troglodytes, A. diaperinus, S. unifasciatus, G. cornutus, Euxestus sp., E. rubriculus, Ontholestes sp. e Dactylosternum sp.

PALAVRAS-CHAVE. Coleoptera, flutuação populacional, aviário.

A ordem Coleoptera, com cerca de 370.000 espécies descritas (PereiRa \& AlmeIDA, 2001), constitui o maior grupo conhecido de insetos, o que corresponde, aproximadamente, a 40\% da Classe Insecta e a 30\% do Reino Animalia (LAwrenCE \& BritTon, 1991). Como foram os primeiros insetos holometábolos a evoluírem, ocuparam grande quantidade de nichos ecológicos (Evans, 1975).

Dentre os sistemas em que podem ser encontrados estão os que foram modificados pelo homem com a finalidade, basicamente, de viabilizar a obtenção de alimento, os referidos agroecossistemas ou ecossistemas artificiais. Em tais ecossistemas, encontram-se os aviários, onde existem diversos artrópodes sinantrópicos e, dentre eles, algumas espécies de coleópteros. Destacam-se em aviários do mundo inteiro Alphitobius diaperinus (Panzer,
1797) (Tenebrionidae), por ser considerada uma das mais importantes pragas que ocorrem em cama de galinha (Axtell, 1985, 1994; AXtell \& ARENDs, 1990), e Carcinops pumilio (Erichson, 1834) (Histeridae), um dos principais artrópodes predadores de dípteros muscóideos que se desenvolvem em fezes de galinhas poedeiras (AxTELL, 1985; Geden \& Stoffolano Jr., 1987; Geden \& Axtell, 1988; Geden et al., 1988; GeDEN, 1990).

Alphitobius diaperinus foi registrada pela primeira vez, em 1950, em aviários dos Estados Unidos. Até então, era conhecida como praga de produtos armazenados (Gould \& Moses, 1951; Harding \& Bissel, 1958). Espécie onívora (HARRIs, 1966; ARENDS, 1987; DesPins et al., 1989) apresenta importância econômica por relacionar-se a doenças importantes na avicultura (CASAS et al., 1972; Axtell, 1994; Steelman, 1996); por constituir alimento 
alternativo para as aves de corte, principalmente entre o $4^{\circ}$ e $21^{\circ}$ dias de vida (MATIAs, 1992), o que pode resultar em diminuição do ganho de peso (AxTEll \& Arends, 1990), além de ocasionar hemorragias no tubo digestivo; por destruir as estruturas de madeira que dão suporte às gaiolas (FERNANDES et al., 1995) e os materiais isolantes, do teto e das paredes, dos aviários de países de clima frio (VAUGHAN et al., 1984; TuRner JR., 1986; Despins et al., 1987; Voris et al., 1994). Aspectos positivos podem ser ressaltados como as alterações físicas provocadas no esterco por suas larvas que, ao fazerem galerias (WILSON \& Miner, 1969), promovem a aeração e a secagem do substrato, inviabilizando, assim, o desenvolvimento de moscas (Despins et al., 1988; Geden, 1990) e a sua capacidade predatória em relação a Musca domestica L., 1758 (Diptera, Muscidae) (Despins et al., 1988) e Corcyra cephalonica (Stainton, 1866) (Lepidoptera, Pyralidae) (DAss et al., 1984).

Carcinops pumilio vem sendo considerado a mais eficiente na redução de populações de $M$. domestica em aviários norte-americanos, devido à sua alta capacidade predatória, distribuição no esterco e abundância (AxTELL, 1986). Segundo Geden \& Axtell (1988), as larvas de $2^{\circ}$ instar e os adultos predam, respectivamente, 26 e 48/49 imaturos de $M$. domestica por dia. Podem alimentar-se de ácaros acarídeos e de outros pequenos dípteros e, por isso, são considerados predadores generalistas (GEDEN, 1990). No Brasil, ocorre Carcinops troglodytes (Paykull, 1811) cuja importância, provavelmente, seja equivalente à da espécie norte-americana, $C$. pumilio. Estas espécies até podem ser encontradas juntas, porém $C$. pumilio ocorre, preferencialmente, em regiões temperadas e $C$. troglodytes, em tropicais (GEDEN, 1990). Carcinops troglodytes é citada por BERTI FILHO et al. (1989) e por MARICONI et al. (1999) como predadora de M. domestica.

Objetiva-se identificar os coleópteros que ocorrem em uma granja avícola em Pelotas, Rio Grande do Sul e estimar a flutuação populacional das espécies mais significativas.

\section{MATERIAL E MÉTODOS}

O trabalho foi conduzido em Pelotas, RS, na granja do Conjunto Agrotécnico Visconde da Graça (CAVG), pertencente à Universidade Federal de Pelotas ( $31^{\circ} 34^{\prime} \mathrm{S}$; $\left.52^{\circ} 23^{\prime} \mathrm{W}\right)$, no período de agosto de 1998 a julho de 1999. A granja, distante $7 \mathrm{~km}$ do centro da cidade, tem capacidade para alojar 14.000 aves. As instalações abrangem uma área de um hectare, onde estão distribuídos nove galpões galvanizados fechados, sendo três para a criação de galinhas poedeiras ou matrizes em cama com maravalhas e seis para criação de poedeiras em gaiola. Cada galpão apresenta uma área de $245 \mathrm{~m}^{2}$ (20 $\mathrm{m}$ de comprimento $\mathrm{x} 12,25 \mathrm{~m}$ de largura) e capacidade para abrigar 1.200 a 3.400 aves, dependendo do tipo de criação. Nas proximidades da granja, há estábulo para bovinos de leite e criação de suínos e coelhos, além do plantio de milho, pastagens e horticultura.

As coletas foram realizadas no galpão de poedeiras em cama com maravalhas, que apresentava um puleiro central ( $14 \mathrm{~m}$ comprimento x $10 \mathrm{~m}$ largura x $0,6 \mathrm{~m}$ altura) formado por muretas de concreto e coberto por um estrado de madeira com tela, no qual eram suspensos os bebedouros e os comedouros. No interior do puleiro, as fezes das aves ficavam retidas durante todo o período de permanência do lote, funcionando como área de refúgio e alimentação de diversas espécies de artrópodes. As aves não tinham acesso às fezes. A área de circulação ao redor do puleiro apresentava uma camada de maravalhas com cerca de $10 \mathrm{~cm}$ de altura, reposta sempre que necessário. O lote contendo 1.075 galinhas da linhagem "Isa Brown" foi instalado neste galpão, em 16.I.1998, com 90 dias de idade, tendo sido descartado em 20.X.1999. Durante todo o período experimental não foi aplicado nenhum produto químico para controle de artrópodes.

Para a coleta dos coleópteros foram utilizados seis métodos. Nos métodos 1 a 4, as bandejas plásticas $(20 \mathrm{x}$ $20 \times 10 \mathrm{~cm}$ ) com tampas eram preenchidas com aproximadamente um quilo de fezes de galinhas (com menos de $24 \mathrm{~h}$ ), com três repetições para cada método. As bandejas dos métodos 1 e 4, com fezes recentes, eram expostas imediatamente, no galpão de poedeiras em cama, enquanto que as bandejas dos métodos 2 e 3 eram isoladas em gaiola, com tela contra insetos, até o momento da exposição (7 e 14 dias, respectivamente). As bandejas do método 4 ficavam expostas durante as três semanas, e as dos métodos 1, 2 e 3, apenas uma semana cada. Devido ao tipo de estrutura deste galpão, as fezes utilizadas nos métodos 1, 2, 3 e 4 foram recolhidas no galpão de poedeiras em gaiolas.

As bandejas com fezes, ao findar o período de exposição no galpão, eram levadas ao laboratório, colocadas dentro dos funis de alumínio $(\varnothing=35 \mathrm{~cm})$, cobertos com toucas de pano e com frascos plásticos transparentes acoplados (coletores com $200 \mathrm{ml}$ de capacidade) contendo álcool $70 \%$, por sete dias, em câmara climatizada a $\pm 25^{\circ} \mathrm{C}$, com U.R. $\geq 80 \%$, para a captura dos coleópteros. Terminado este período, os frascos coletores eram substituídos por outros e lâmpadas ( $40 \mathrm{~W}$ ) eram colocadas incidindo sobre cada funil, por mais quatro dias.

O método 5 consistiu na coleta de três amostras com cerca de um quilo de fezes acumuladas sob o estrado do galpão (de poedeiras), posteriormente acondicionadas em bandejas e, encaminhadas ao laboratório. No laboratório, as etapas cumpridas para obtenção dos coleópteros foram as mesmas executadas para os métodos 1 a 4. Em complementação, foi utilizado o método da Flotação (ToBın \& PITTs, 1999), colocando-se pequenas quantidades de fezes, em uma bandeja plástica branca $(29 \times 34 \times 5 \mathrm{~cm})$ contendo água, para remoção dos coleópteros. Previamente, as amostras eram colocadas no congelador, onde permaneciam por 24 horas para sacrificar os insetos.

No método 6 foi utilizada armadilha conhecida como "Tube Trap", idealizada por J. J. Arends e descrita por SAFrit \& Axtell (1984). Esta armadilha consiste de um tubo de PVC com $4 \mathrm{~cm}$ de diâmetro e $17 \mathrm{~cm} \mathrm{de}$ comprimento, dentro do qual insere-se um papelão corrugado, com $17 \mathrm{~cm}$ de largura por $25 \mathrm{~cm} \mathrm{de}$ comprimento, enrolado em espiral (de forma que as ondulações fiquem no sentido longitudinal do tubo). Oito armadilhas foram dispostas pelo chão do galpão, junto à mureta de concreto, fixadas com tijolos e cobertas com maravalhas para evitar o movimento das mesmas pelo 
esgravatar das galinhas. Para o monitoramento dos coleópteros foram realizadas duas visitas por mês: uma até o dia dez e a outra após o dia vinte. A cada visita, as armadilhas eram recolhidas e examinadas separadamente, removendo os coleópteros para posterior identificação e contagem. Após este procedimento, as armadilhas eram recolocadas em seus respectivos locais.

Para a identificação dos coleópteros, foram utilizados os trabalhos de ARNETT (1963), Gorham (1991), Haines (1991), LaWrence \& BritTon (1991), Mound (1989) e Pereira \& Almeida (2001). O material testemunho encontra-se depositado na Coleção Entomológica "Pe. Jesus Santiago Moure", do Departamento de Zoologia, da Universidade Federal do Paraná, sob responsabilidade da Dra Luciane Marinoni, curadora do acervo.

A flutuação populacional foi estimada tomando-se as observações, referentes aos seis métodos de coleta, previamente transformadas em $\sqrt{x+0,5}$, como repetição e utilizando-se o método da análise de regressão polinomial.

\section{RESULTADOSE DISCUSSÃO}

Durante o período de estudo, foram capturados 46.715 artrópodes, dos quais 12.449 (26,6\%) corresponde aos coleópteros Carcinops troglodytes; Alphitobius diaperinus; Somotrichus unifasciatus (Dejean, 1792); Gnathocerus cornutus (Fabricius, 1798); Euxestus sp.; Euspilotus rubriculus (Marseul, 1855); Ontholestes sp.; Dactylosternum sp.; Mezium americanum (Laporte, 1840); Palorus subdepressus (Wollaston, 1864); Tenebroides mauritanicus (L., 1758), Xyleborus ferrugineus (Fabricius, 1801) e espécimens não identificados de Dermestidae (tab. I).
Ao longo dos doze meses de coleta, houve variação mensal no número de espécies capturadas, tendo ocorrido, em março e julho, o maior número (12) e em setembro, o menor (7). Março foi quando se registrou a maior temperatura média mensal $\left(23,3^{\circ} \mathrm{C}\right)$, enquanto que setembro apresentou uma das temperaturas médias mensais mais baixas de todo o período amostral $\left(14^{\circ} \mathrm{C}\right)$ (fig. 1, tab. II). A presença de coleópteros foi observada ao longo de todo o ano, porém foi em março que se

Tabela I. Frequiência de captura das espécies de Coleoptera em galpão de aves poedeiras, no período de agosto de 1998 a julho de 1999, em Pelotas, RS (FA, Freqüência absoluta; FR, Freqüência relativa).

\begin{tabular}{lrr}
\hline Espécies (Famílias) & FA $\left(\mathrm{n}^{\circ}\right)$ & FR $(\%)$ \\
\hline Carcinops troglodytes (Histeridae) & 6444 & 13,795 \\
Alphitobius diaperinus (Tenebrionidae) & 2896 & 6,200 \\
Somotrichus unifasciatus (Carabidae) & 1190 & 2,547 \\
Gnathocerus cornutus (Tenebrionidae) & 947 & 2,027 \\
Euxestus sp. (Cerylonidae) & 394 & 0,843 \\
Euspilotus rubriculus (Histeridae) & 213 & 0,456 \\
Ontholestes sp. (Staphylinidae) & 190 & 0,407 \\
Dactylosternum sp. (Hydrophilidae) & 93 & 0,199 \\
Mezium americanum (Ptinidae) & 43 & 0,092 \\
Palorus subdepressus (Tenebrionidae) & 27 & 0,058 \\
Tenebroides mauritanicus (Trogossitidae) & 8 & 0,017 \\
Xyleborus ferrugineus (Scolytidae) & 1 & 0,002 \\
Espécimens não identificados de Dermestidae & 3 & 0,006 \\
Outros insetos & 29964 & 64,142 \\
Outros artrópodes & 4302 & 9,209 \\
\hline Total & 46715 & 100,000 \\
\hline
\end{tabular}

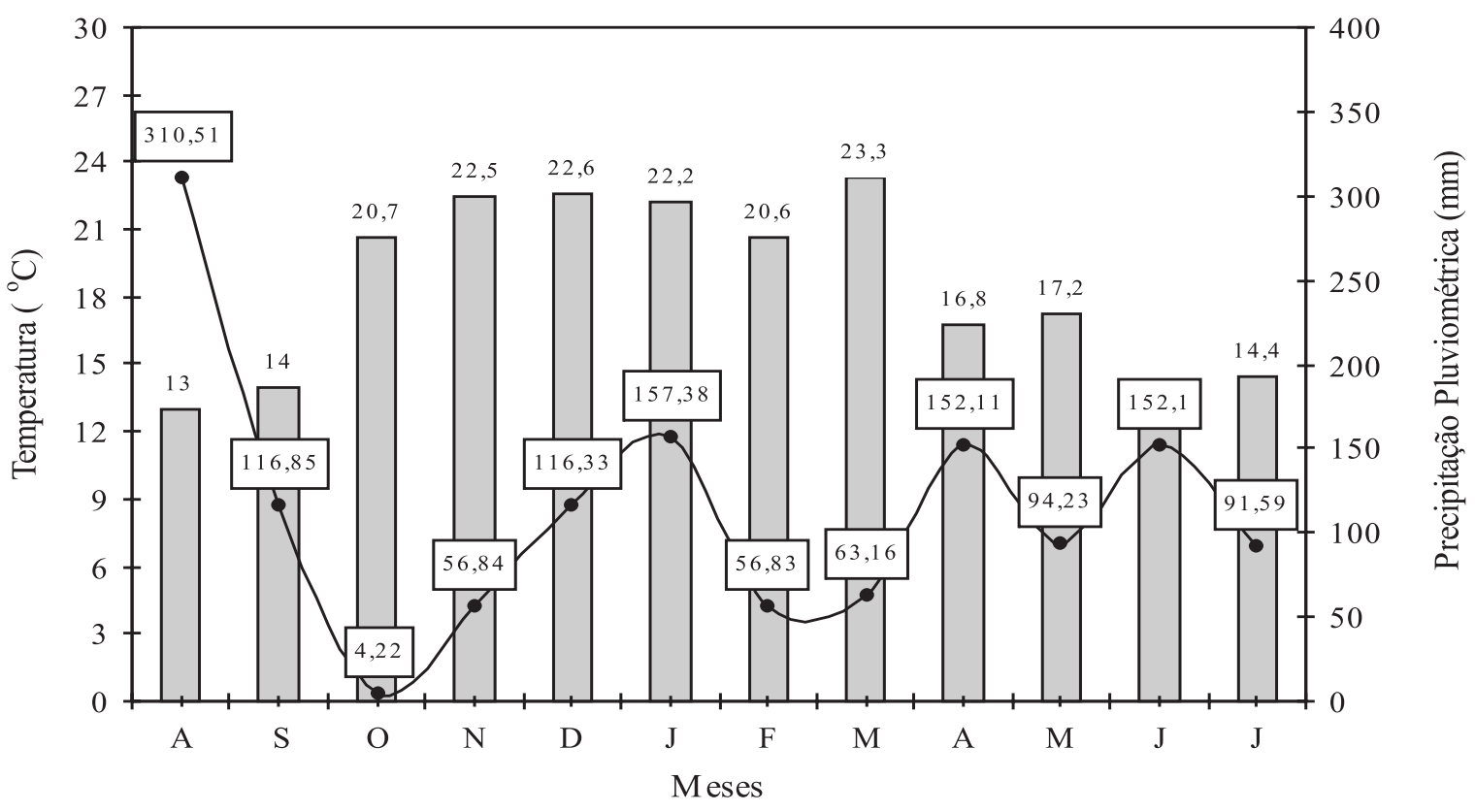

Temperatura $\longrightarrow$ Precipitação pluviométrica

Fig. 1. Temperatura média mensal e precipitação pluviométrica, no período de agosto de 1998 a julho de 1999 , em Pelotas, RS (Estação Climatológica do Conjunto Agrotécnico Visconde da Graça). 
Tabela II. Espécies de Coleoptera capturadas em galpão de aves poedeiras, no período de agosto de 1998 a julho de 1999, em Pelotas, RS.

\begin{tabular}{|c|c|c|c|c|c|c|c|c|c|c|c|c|c|}
\hline \multirow{2}{*}{ Famílias/Espécies } & \multicolumn{5}{|c|}{1998} & \multicolumn{8}{|c|}{1999} \\
\hline & Ago & Set & Out & Nov & Dez & Jan & Fev & Mar & Abr & Maio & Jun & Jul & Total \\
\hline \multicolumn{14}{|l|}{ Carabidae } \\
\hline Somotrichus unifasciatus & 90 & 66 & 40 & 82 & 195 & 222 & 112 & 153 & 94 & 44 & 67 & 25 & 1190 \\
\hline \multicolumn{14}{|l|}{ Hydrophilidae } \\
\hline Dactylosternum sp. & 3 & & 25 & 13 & & 5 & 12 & 3 & 14 & 12 & 2 & 4 & 93 \\
\hline \multicolumn{14}{|l|}{ Histeridae } \\
\hline Carcinops troglodytes & 728 & 575 & 294 & 444 & 491 & 387 & 442 & 672 & 1063 & 340 & 606 & 402 & 6444 \\
\hline Euspilotus rubriculus & 2 & 4 & 1 & 1 & 7 & 19 & 12 & 22 & 41 & 40 & 32 & 32 & 213 \\
\hline \multicolumn{14}{|l|}{ Staphylinidae } \\
\hline Ontholestes sp. & 1 & 7 & 48 & 53 & 13 & 26 & 22 & 5 & 06 & 1 & 6 & 2 & 190 \\
\hline \multicolumn{14}{|l|}{ Dermestidae } \\
\hline Espécimens não identificados & 1 & 1 & & & & & & & & & & 1 & 3 \\
\hline \multicolumn{14}{|l|}{ Ptinidae } \\
\hline Mezium americanum & & & & & 12 & 4 & 3 & 8 & 6 & 2 & 6 & 2 & 43 \\
\hline \multicolumn{14}{|l|}{ Trogossitidae } \\
\hline Tenebroides mauritanicus & & & & & & & & 3 & 4 & & & 1 & 8 \\
\hline \multicolumn{14}{|l|}{ Cerylonidae } \\
\hline Euxestus sp. & 7 & 17 & 3 & 9 & 10 & 83 & 17 & 48 & 87 & 15 & 17 & 81 & 394 \\
\hline \multicolumn{14}{|l|}{ Tenebrionidae } \\
\hline Alphitobius diaperinus & 31 & 127 & 221 & 18 & 380 & 371 & 313 & 976 & 137 & 139 & 114 & 69 & 2896 \\
\hline Gnathocerus cornutus & 12 & & 1 & 13 & 56 & 103 & 159 & 249 & 190 & 117 & 19 & 28 & 947 \\
\hline Palorus subdepressus & & & & 1 & & & & 19 & 1 & 1 & 1 & 4 & 27 \\
\hline
\end{tabular}

Scolytidae

\begin{tabular}{lccccccccccccc} 
Xyleborus ferrugineus & \multicolumn{10}{c}{1} & \multicolumn{10}{c}{1} \\
\hline Número de espécies & 9 & 7 & 8 & 9 & 8 & 9 & 9 & 12 & 11 & 10 & 10 & 12 & \\
\hline Número de indivíduos & 875 & 797 & 633 & 634 & 1164 & 1220 & 1092 & 2159 & 1643 & 711 & 870 & 651 & 12449
\end{tabular}

assinalou o maior número de indivíduos capturados (2.159), enquanto que o menor índice de coleta foi registrado em outubro (633) (fig. 2, tab. II). Foram capturados $41,6 \%$ dos coleópteros na faixa de temperatura média mensal entre $20,8^{\circ} \mathrm{C}$ e $23,3^{\circ} \mathrm{C}$ (tab. III). Pela análise de regressão, o modelo cúbico, $\mathrm{y}=33,347-6,027 \mathrm{X}+$ $1,630 X^{2}-0,100 X^{3}$ foi significativo $(6,5 \%)$, com coeficiente de determinação de $56,4 \%$.

Carcinops troglodytes (Histeridae) foi a espécie mais abundante, com 6.444 espécimens coletados, representando $51,8 \%$ da ordem (tab. II). Resultados similares foram obtidos por BRUNO et al. (1993), que assinalaram sua ocorrência em todas as granjas amostradas dos diversos municípios do Estado de São Paulo, perfazendo $31,6 \%$ dos coleópteros coletados. Gianizella \& PRADO (1998), em levantamento das espécies de histerídeos em granja em Monte Mor, SP, encontraram sete espécies ocorrendo naquele ambiente e dentre elas, C. troglodytes (33\% dos 19.668 histerídeos capturados). A presença deste coleóptero em excrementos de galinhas, bovinos e suínos foi registrada por LEGNER (1965), LEGNER \& OLTON (1970) e LEGNER et al. (1981) em localidades como Porto Rico, Costa Rica, Samoa Americana e Uganda. Durante o período de amostragem, $C$. troglodytes esteve presente em todos os meses, tendo sido assinalado o acme populacional em abril, quando foram coletados 1.063 espécimens. O menor índice de captura foi registrado em outubro (294) (fig. 3, tab. II). Carcinops troglodytes teve sua maior freqüência nos intervalos de temperaturas médias mensais de $12,4^{\circ} \mathrm{C}$ a $15,2^{\circ} \mathrm{C}(35,9 \%)$ e $20,8^{\circ} \mathrm{C}$ a $23,3^{\circ} \mathrm{C}(30,9 \%)$ (tab. III). O modelo que melhor se ajustou aos dados foi o cúbico, $\mathrm{y}=34,499-8,877 \mathrm{X}+1,609 \mathrm{X}^{2}$ $0,081 \mathrm{X}^{3}$, significativo a $9,8 \%$, com coeficiente de determinação de $38,2 \%$

Alphitobius diaperinus (Tenebrionidae) foi a segunda espécie mais capturada $(23,3 \%)$, tendo ocorrido durante todo o ano (tab. II). Este resultado está de acordo com o obtido por PFeIFFER \& Axtell (1980) que, ao relacionarem cerca de 120 espécies de Coleoptera em

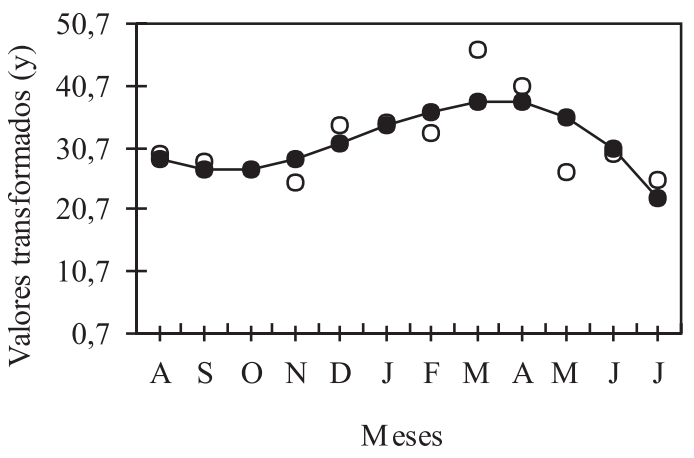

○ Valores transformados $\longrightarrow$ - Valores estimados

Fig. 2. Flutuação populacional das espécies de Coleoptera, em granja avícola, no período de agosto de 1998 a julho de 1999, em Pelotas, RS. 
Tabela III. Distribuição de freqüências de captura das espécies de Coleoptera, de acordo com a temperatura média mensal, na granja Colégio Agrotécnico Visconde da Graça, Pelotas, RS, no período de agosto de 1998 a julho de 1999, (Abs., Absoluta; Rel., Relativa; ¿, somatório das 13 espécies capturadas).

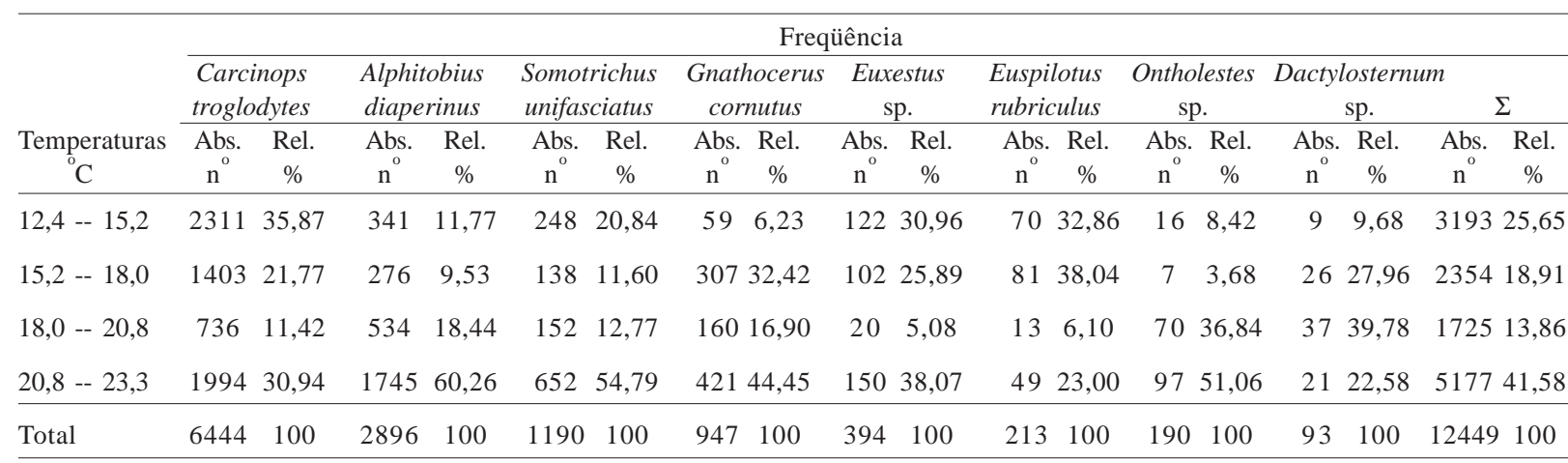

granjas de três regiões da Carolina do Norte, mencionaram este coleóptero como o segundo mais abundante (11.521), antecedido por Carcinops pumilio (42.003). Conforme Fernandes et al. (1995), em Uberlândia, MG, A. diaperinus foi mais abundante na granja estudada, perfazendo 1.713 dos 3.011 coleópteros capturados. BRUNo et al. (1993), em diversos municípios do Estado de São Paulo, registraram A. diaperinus como o terceiro coleóptero mais abundante nos aviários em questão. Sua presença foi assinalada por LEGNER \& OLTON (1970) em excrementos de galinhas (Califórnia, Israel, Samoa Americana), de bovinos (Califórnia, Porto Rico, Israel), de eqüinos (Israel) e de suínos (Samoa Americana) e por LomônACO \& PRADO (1994), em excremento de galinhas, em Uberlândia, MG. O maior índice de captura de $A$. diaperinus foi registrado em março (976) (fig. 4, tab. II). A menor ocorrência foi assinalada em novembro (18) (tab. II). Estes resultados são corroborados aos encontrados por Chernaki \& Almeida (2001) que, ao criarem estes insetos para obtenção das exigências térmicas, indicaram que ovos, larvas e pupas apresentaram maior sobrevivência nas temperaturas mais elevadas (entre $28^{\circ} \mathrm{C}$ e $\left.31^{\circ} \mathrm{C}\right)$. Esta espécie ocorreu em maior frequiência $(60,3 \%)$ na faixa de temperatura média mensal entre $20,8^{\circ} \mathrm{Ce} 23,3^{\circ} \mathrm{C}$ (tab. III). O modelo ajustado foi o quadrático, $\mathrm{y}=-0,654$ $+5,762 X-0,423 X^{2}$, com significância de $5,7 \%$ e com coeficiente de determinação de $41,8 \%$.

A alta ocorrência de Carcinops troglodytes e Alphitobius diaperinus pode ser atribuída à similaridade existente entre o substrato encontrado na granja e o habitat originário destas espécies, ninhos de pássaros selvagens na África (Gould \& Moses, 1951; HARDING JR. \& Bissel, 1958; GEDEN, 1990).

Somotrichus unifasciatus (Carabidae) ocorreu em todos os meses amostrados, sendo que o pico populacional foi registrado em janeiro (222). Julho foi o mês de menor ocorrência (25) (fig. 5, tab. II). O intervalo de temperatura média mensal em que se capturou a maioria de $S$. unifasciatus foi entre $20,8^{\circ} \mathrm{C}$ e $23,3^{\circ} \mathrm{C}(54,8 \%$ ) (tab. III). O modelo que melhor se ajustou aos dados foi o de $4^{\circ}$ grau, $y=15,942-9,437 X+3,305 X^{2}-0,388 X^{3}+0,014 X^{4}$, e a análise de regressão polinomial realizada identificou significância de $5,4 \%$, com coeficiente de determinação de $73,8 \%$.

Gnathocerus cornutus (Tenebrionidae) foi coletada em 11 dos 12 meses de amostragem, não havendo captura em setembro (fig. 6, tab. II). O acme populacional aconteceu em março (249) (tab. II) e o menor índice de captura foi assinalado em outubro (1) (tab. II). Esta espécie foi coletada por BRUNo et al. (1993) em granjas do Estado de São Paulo, porém numa quantidade menor, ou seja, somente 9 espécimens. O acme populacional para $G$. cornutus foi registrado na faixa de temperatura média mensal de $20,8^{\circ} \mathrm{C}$ a $23,3^{\circ} \mathrm{C}(44,5 \%)$ (tab. III). O modelo ajustado foi o cúbico, $\mathrm{y}=4,576-3,536 \mathrm{X}+1,182 \mathrm{X}^{2}-$ $0,075 \mathrm{X}^{3}$, significativo a $0,63 \%$ e com coeficiente de determinação de $85,9 \%$.

Euxestus sp. (Cerylonidae) apresentou acme populacional em abril (87) e menor índice de coleta em outubro (3) (fig. 7, tab. II). Estes resultados diferem dos de BRUNO et al. (1993), que coletaram somente dois espécimens de uma espécie não identificada de Euxestus sp., em uma granja de São Paulo. Os maiores índices de captura para Euxestus sp. foram assinalados no intervalo de temperatura média mensal de $12,4^{\circ} \mathrm{C}$ a $18,0^{\circ} \mathrm{C}(56,9 \%)$ (tab. III). O modelo de regressão ajustado foi o linear, y = $2,404+0,4241 \mathrm{X}$, significativo a $6,7 \%$ e com coeficiente de determinação de $31,9 \%$.

O coleóptero Euspilotus rubriculus (Histeridae) ocorreu em todos os meses amostrados, sendo que seu acme populacional foi registrado em abril (41). A menor ocorrência foi assinalada nos meses de outubro e novembro (1) (fig. 8, tab. II). A presença de algumas espécies de Euspilotus Lewis, 1907 foi citada por LEGNER \& Olton (1970), ocorrendo em excrementos diversos, como E. liticolus (galinhas e eqüinos, sul da Califórnia e bovinos, sul do México e sul da Califórnia), E. scrupularis (Le Conte, 1860) (galinhas e eqüinos, sul da Califórnia; bovinos, Cottowood e Tucson (Arizona) e Las Cruces (Novo México)), E. mormonellus (Casey, 1924) (bovinos, Cottowood, Arizona) e Euspilotus sp. (bovinos, sul do México); por Pfeiffer \& Axtell (1980), E. (Hesperosaprinus) assimilis (Paykull, 1811), E. (Neosaprinus) scrupularis (Le Conte, 1860) e E. (Hesperosaprinus) sp., em excrementos de galinhas, em três regiões da Califórnia; por Harris \& Blume (1986), que comentaram a existência de uma espécie de Euspilotus associada a excrementos de bovinos, nos Estados Unidos; por FINCHER (1990), que mencionou a existência de três espécies de Euspilotus associadas a 

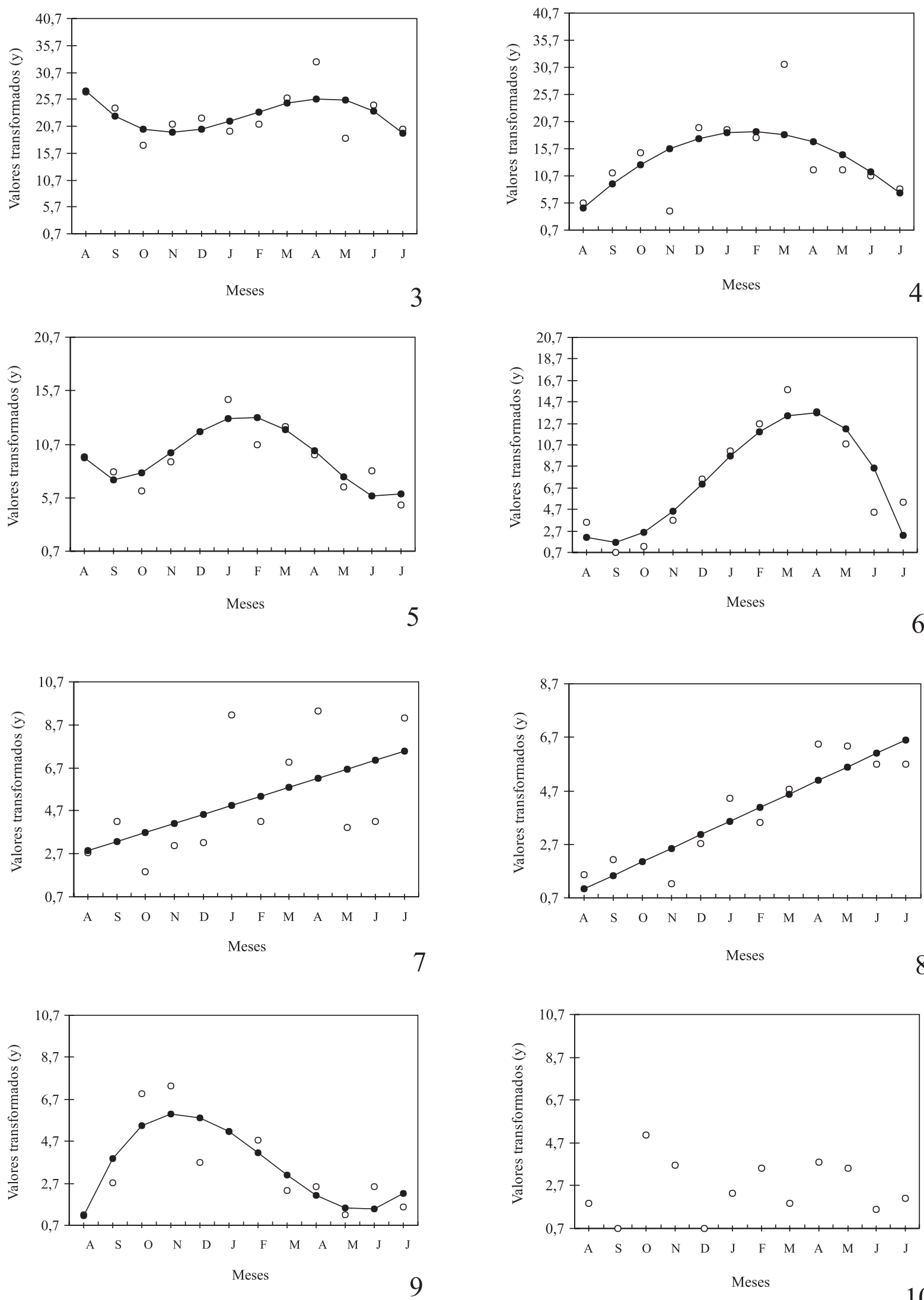

Figs. 3-10. Flutuação populacional das espécies de Coleoptera, em granja avícola, no período de agosto de 1998 a julho de 1999 , em Pelotas, RS. 3, Carcinops troglodytes (Histeridae); 4, Alphitobius diaperinus (Tenebrionidae); 5, Somotrichus unifasciatus (Carabidae); 6, Gnathocerus cornutus (Tenebrionidae); 7, Euxestus sp. (Cerylonidae); 8, Euspilotus rubriculus (Histeridae); 9, Ontholestes sp. (Staphylinidae); 10, Dactylosternum sp. (Hydrophilidae) (círculo branco: valores transformados; círculo preto: valores estimados). 
excrementos de bovinos, nos Estados Unidos; por BRUNO et al. (1993), E. modestus e E. (Neosaprinus) sp., em excrementos de galinhas, em diversos municípios do Estado de São Paulo; por Fernandes et al. (1995), Euspilotus sp., em excrementos de galinhas, em Uberlândia, MG e por Gianizella \& Prado (1998), E. modestus e E. (Hesperosaprinus) sp., em excrementos de galinhas, em Monte Mor, SP. Euspilotus rubriculus teve seu acme populacional na faixa de temperatura média mensal entre $12,4^{\circ} \mathrm{C}$ e $18,0^{\circ} \mathrm{C}(70,9 \%)$ (tab. III). O modelo ajustado foi linear, $\mathrm{y}=0,527+0,506 \mathrm{X}$, significativo a $0,47 \%$, com coeficiente de determinação de $82,8 \%$.

Ontholestes sp. (Staphylinidae) foi capturado durante todo o período amostral, tendo o seu acme populacional assinalado em novembro (53). Os meses de agosto e maio destacaram-se pelo menor índice de captura (1) (fig. 9, tab. II). O. cingulatus (Gravenhorst, 1802) foi mencionada por SANDERS \& DOBSON (1966) ocorrendo em fezes bovinas, em Indiana. Ontholestes sp. foi capturado em maior número no intervalo de temperatura média mensal de $20,8^{\circ} \mathrm{C}$ a $23,3^{\circ} \mathrm{C}(51,1 \%)$ (tab. III). Pela análise de regressão polinomial, o modelo cúbico, $\mathrm{y}=-2,925+$ $4,873 \mathrm{X}-0,806 \mathrm{X}^{2}+0,036 \mathrm{X}^{3}$, foi significativo a $2,9 \%$, com coeficiente de determinação de $73,7 \%$.

Dactylosternum sp. (Hydrophilidae), ao longo do período experimental, esteve presente em 10 dos 12 meses amostrados, não havendo captura em setembro e dezembro. O maior índice de coleta foi realizado em outubro (25) e o menor, em junho (2) (fig. 10, tab. II). RUEDA et al. (1990) assinalaram a presença de diversos predadores associados à granja avícola, na região das Filipinas e, dentre eles, citaram a ocorrência de $D$. abdominale (Fabricius, 1792). Dactylosternum sp. apresentou os maiores índices de captura na faixa de temperatura média mensal de $15,2^{\circ} \mathrm{C}$ a $23,3^{\circ} \mathrm{C}(90,3 \%)$ (tab. III). A análise de regressão polinomial realizada não identificou modelo significativo para a flutuação populacional desta espécie.

Embora o tipo de galpão utilizado no experimento apresentasse uma estrutura diferenciada, existiu uma considerável variação de temperatura que afetou a flutuação populacional dos artrópodes ali presentes. Segundo DAJOZ (1983), os animais ectotérmicos são muito mais dependentes dos fatores climáticos quando comparados aos endotérmicos. Entretanto, além destes, outros fatores estariam interagindo nessa flutuação, como os exógenos e os endógenos, que incluem, respectivamente, os fatores dependentes da densidade (i.e., disponibilidade de alimento, espaço, predação) e os fatores independentes da densidade (i.e., clima, componentes genéticos, interação social, dispersão) (Pinto-Coelho, 2000).

As espécies de coleópteros comentadas a seguir não tiveram suas flutuações populacionais estimadas devido às baixas densidades populacionais e freqüências mensais. Foram capturados 43 espécimens de Mezium americanum (Ptinidae) no período de dezembro a julho, sendo dezembro o mês de maior captura (12) (tab. II).

Um total de 27 espécimens de Palorus subdepressus (Tenebrionidae) foi coletado no mês de novembro e nos meses de março a julho (tab. II). Em março foi assinalado o maior índice de captura (19) (tab. II), coincidindo com a temperatura média mensal máxima $\left(23,3^{\circ} \mathrm{C}\right)$ obtida durante o período experimental (fig. 1). Esta espécie foi capturada por Pfeiffer \& Axtell (1980) em aviários na Carolina do Norte. Tenebroides mauritanicus (Trogossitidae) teve sua presença assinalada nos meses de março (3), abril (4) e julho (1) (tab. II).

Em março, foi registrada a captura de um Xyleborus ferrugineus (Scolytidae) (tab. II). Este grupo de insetos é encontrado em florestas e, provavelmente, sua ocorrência nesse agroecossistema seja acidental. Entretanto, PFEIFFER \& Axtell (1980) também coletaram uma espécie de Xyleborus, X. saxeseni (Ratzeburg, 1837), em aviários na Carolina do Norte. Espécimens não identificados de Dermestidae foram capturados nos meses de agosto (1), setembro (1) e julho (1) (tab. II).

Agradecimentos. Ao CNPq, pela concessão da bolsa; ao Departamento de Microbiologia e Parasitologia e ao Conjunto Agrotécnico Visconde da Graça (UFPel), por permitirem a realização deste trabalho em suas instalações; ao médico veterinário Paulo Renato Peixoto Costa (UFPel) (in memoriam), por sua colaboração nas saídas de campo e na manutenção do laboratório e aos especialistas Dr. John Mark Kingsolver (Florida State Collection of Arthropods) (Cerylonidae e Hydrophilidae), Dr. Nicolas Degallier (EMBRAPA) e Dr. Gerardo Arriagada (Profissional autônomo, Santiago do Chile) (Histeridae), Dr. Carlos Flechtmann (FEIS/UNESP) (Scolytidae) pelo valioso auxílio na identificação do material.

\section{REFERÊNCIASBIBLIOGRÁFICAS}

Arends, J. J. 1987. Control, management of the litter beetle. Poultry Digest (April):173-176.

Arnett, R. H. 1963. The beetles of the United States: a manual for identification. Washington, The Catholic University of America. $1112 \mathrm{p}$.

Axtell, R. C. 1985. Arthropod pests of poultry. In: Williams, R. E.; Hall, R. D.; Broce, A. B. \& Scholl, P. J. eds. Livestock Entomology. New York, Willey Interscience. p.269-295.

1986. Fly management in poultry production: cultural, biological, and chemical. Poultry Science 65:657-667. 1994. Litter beetles and other poultry premise pests. Kansas, Miles. 10 p.

Axtell, R. C. \& ARENDS, J. J. 1990. Ecology and management of arthropod pests of poultry. Annual Review of Entomology 35:101-126.

Berti Filho, E.; Costa, V. A. \& Aagesen, T. L. 1989. Occurrence of natural enemies of Musca domestica L. (Diptera: Muscidae) in poultry areas of Bastos, State of São Paulo, Brazil. Revista de Agricultura 64(1): 98-98.

Bruno, T. V.; Guimarães, J. H.; Santos, A. M. M. dos \& Tucci, E. C. 1993. Moscas sinantrópicas (Diptera) e seus predadores que se criam em esterco de aves poedeiras confinadas, no Estado de São Paulo, Brasil. Revista Brasileira de Entomologia 37(3):577-590.

Casas, E. de Las; Harein, P. K. \& Pomeroy, B. S. 1972. Bacteria e fungi within the lesser mealworm collected from poultry brooder houses. Environmental Entomology 1(1):27-30.

Chernaki, A. M. \& Almeida, L. M. 2001. Exigências térmicas, período de desenvolvimento e sobrevivência de imaturos de Alphitobius diaperinus (Panzer) (Coleoptera: Tenebrionidae). Neotropical Entomology 30(3):365-368.

Dajoz, R. 1983. Ecologia Geral. Petrópolis, Vozes. 472 p.

Dass, R.; Navarajan Paul, A. V. \& Agarwal, R. A. 1984. Feeding potencial and biology of lesser mealworm, Alphitobius diaperinus (Panz.) (Col., Tenebrionidae), preying on Corcyra cephalonica St. (Lep., Pyrallidae). Journal of Applied Entomology 98(5):445-447.

Despins, J. L.; Turner, E. C., JR. \& Ruszler, P. L. 1987. 
Construction profiles of high rise caged layer houses in association with insulation damage caused by the lesser mealworm, Alphitobius diaperinus (Panzer) in Virginia. Poultry Science 66:243-250.

Despins, J. L.; Turner, E. C. JR. \& Ruszler, P. L. 1989. Effects of poultry manure moisture and poultry house construction materials on the movements of the lesser mealworm, Alphitobius diaperinus (Panzer) (Coleoptera: Tenebrionidae), a structural insect pest in high rise caged layer houses. Poultry Science 68:1326-1331.

Despins, J. L.; Vaughan, J. A. \& Turner, E. C. JR. 1988. Role of the lesser mealworm, Alphitobius diaperinus (Panzer) (Coleoptera: Tenebrionidae), as a predator of the house fly, Musca domestica L. (Diptera: Muscidae), in poultry houses. The Coleopterists Bulletin 42(3):211-216.

Evans, G. 1975. The Life of Beetles. London, George Allen \& Unwin. $105 \mathrm{p}$.

Fernandes, M. A.; Santos, M. A. S. \& Lomônaco, C. 1995. Ocorrência de artrópodes no esterco acumulado em uma granja de galinhas poedeiras. Anais da Sociedade Entomológica do Brasil 24(3):649-654.

Fincher, G. T. 1990. Biological control of dung-breeding flies: pests of pastured cattle in the United States. In: RutZ, D. A. \& PAtTerson, R. S. eds. Biocontrol of arthropods affecting livestock and poultry. Boulder, Westview. p.137-151.

Geden, C. J. 1990. Coleoptera and acarine predators of house fly immatures in poultry production systems. In: RuTz, D. A. \& PAtTERSON, R. S. eds. Biocontrol of arthropods affecting livestock and poultry. Boulder, Westview. p.177-200.

Geden, C. J. \& Axtell, R. C. 1988. Predation by Carcinops pumilio (Coleoptera: Histeridae) and Macrocheles muscaedomesticae (Acarina: Macrochelidae) on the house fly (Diptera: Muscidae): functional response, effects of temperature, and availability of alternative prey. Environmental Entomology 17(4):739-744.

Geden, C. J.; Stinner, R. E. \& Axtell, R. C. 1988. Predation by predators of the house fly in poultry manure: effects of predator density, feeding history, interspecific interference, and field conditions. Environmental Entomology 17(2):320-329.

Geden, C. J. \& Stoffolano, J. G., JR. 1987. Succession of manure arthropods at a poultry farm in Massachusetts, USA, with observations on Carcinops pumilio (Coleoptera: Histeridae) sex ratios, ovarian condition, and body size. Journal of Medical Entomology 24(2):212-220.

Gianizella, S. L. \& Prado, A. P. 1998. Levantamento e sazonalidade de coleópteros (Histeridae) em criação de aves poedeiras. Anais da Sociedade Entomológica do Brasil 27(4):551-557.

Gorham, J. R., ed. 1991. Insect and mite pests in food. An illustrated key. Washington, Agriculture Handbook $n^{\circ} 655$ v. $1,2,767 \mathrm{p}$

Gould, G. E. \& Moses, H. E. 1951. Lesser mealworm infestation in a brooder house. Journal Economic Entomology 44:265265.

HAINES, C. P. 1991. Insects and arachnids of tropical stored products: their biology and identification. 2.ed., Chatham, Natural Resources Institute. 246p.

Harding W. C., JR. \& Bissell, T. L. 1958. Lesser mealworms in a brooder house. Journal of Economic Entomology 51(1):112-112.

HARRIS, F. 1966. Observations of the lesser mealworm, Alphitobius diaperinus (Panzer). Journal of Georgia Entomology Society 1:17,18.

Harris, R. L. \& Blume, R. R. 1986. Beneficial arthropods inhabiting bovine droppings in United States. Miscellaneous Publications of the Entomological Society of America
61:10-15.

Lawrence, J. F. \& Britton, E. B. 1991. Coleoptera. In: CSIRO ed. The Insects of Australia. 2.ed. Victoria, Melbourne University. v. 2, p.543-683.

LEGNER, E. F. 1965. Un complejo de los artropodos que influyen en los estadios juveniles de Musca domestica L. en Puerto Rico. Caribbean Journal of Science 5(3-4):109-115.

Legner, E. F. \& Olton, G. S. 1970. Worlwide survey and comparison of adult predator and scavenger insect populations associated with domestic animal manure where livestock is artificially congregated. Hilgardia 40(9):225-266.

Legner, E. F.; Greathead, D. J. \& Moore, I. 1981. Equatorial East African predatory and scavenger arthropods in bovine excrement. Environmental Entomology 10:620-625.

Lomônaco, C. \& Prado, A. P. 1994. Estrutura comunitária e dinâmica populacional da fauna de dípteros e seus inimigos naturais em granjas avícolas. Anais da Sociedade Entomológica do Brasil 23(1):71-80.

Mariconi, F. A. M.; Guimarães, J. H. G.; Berti Filho, E. 1999. A mosca doméstica e algumas outras moscas nocivas. Piracicaba, Fundação de Estudos Agrários Luiz de Queiroz. $135 \mathrm{p}$.

Matias, R. S. 1992. Controle de Alphitobius diaperinus em piso e cama de aviários. Pesquisa Agropecuária Brasileira 27(1):205-207.

Mound, L. 1989. Common insect pests of stored food products: a guide to their identification. 7. ed. London, British Museum (Natural History). 68 p.

Pereira, P. R. V. S. \& Almeida, L. M. 2001. Chaves para identificação dos principais Coleoptera (Insecta) associados com produtos armazenados. Revista Brasileira de Zoologia 18(1):271-283.

Pfeiffer, D. G. \& Axtell, R. C. 1980. Coleoptera of poultry manure in caged-layer houses in North Carolina. Environmental Entomology 9:21-28.

Pinto-Coelho, R. M. 2000. Fundamentos em ecologia. Porto Alegre, Artmed. 247p.

Rueda, L. M.; Hugo, C. T. \& Zipagan, M. B. 1990. Filth flies and their potential natural enemies in poultry production systems in the Philippines. In: Rutz, D. A. \& Patterson, R. S. eds. Biocontrol of arthropods affecting livestock and poultry. Boulder, Westview. p. 121-135.

SAfrit, R. D. \& Axtell, R. C. 1984. Evaluations of sampling methods for darkling beetles (Alphitobius diaperinus) in the litter of turkey and broiler houses. Poultry Science 63(12):2368-2375.

Sanders, D. P. \& Dobson, R. C. 1966. The insect complex associated with bovine manure in Indiana. Annals of the Entomological Society of America 59:955-959.

Steelman, D. 1996. Darkling beetles are costly pests. Poultry Digest (October):22,23.

Tobin, P. C. \& Pitts, C. W. 1999. Flotation method for extracting insects from poultry manure samples. Journal of Medical Entomology 36(1):121-123.

Turner, E. C., JR. 1986. Structural and litter pests. Poultry Science, 65:644-648.

Vaughan, J. A.; Turner, E. C., Jr. \& Ruszler, P. L. 1984. Infestation and damage of poultry house insulation by the lesser mealworm, Alphitobius diaperinus. Poultry Science 63(6): 1094-1100.

Voris, J. C.; Meyer, J. A.; Pfost, R. \& Woodbury, R. 1994. Temperature affects lesser mealworm populations in turkey brooder houses. California Agriculture 48(2):18-21.

Wilson, T. H. \& MineR, F. D. 1969. Influence of temperature on development of the lesser mealworm, Alphitobius diaperinus (Coleoptera: Tenebrionidae). Journal of Kansas Entomology Society 42:294-303. 